\title{
Understanding sentences with relative clauses
}

\author{
DAVID T. HAKES, JUDITH S. EVANS and LINDA L. BRANNON \\ University of Texas, Austin, Texas 78712
}

\begin{abstract}
Sentences containing self-embedded relative clauses are generally believed to be difficult to understand because such clauses interrupt the clauses in which they are embedded. However, the experiments that purport to have demonstrated this have confounded the self-embedded or right-branching location of the relative clauses with their internal structure, comparing self-embedded object relatives with right-branching subject relatives. In order to break this confounding, Experiment I compared the comprehension difficulty of self-embedded and right-branching cbject relative clauses on two measures of comprehension difficulty. Experiment II made the same self-embedded vs. right-branching comparison for subject relative clauses. The results of both experiments consistently failed to support the interruption hypothesis.
\end{abstract}

During the past decade, sentences with self-embedded relative clauses like Sentence 1 , taken from Miller and Isard (1964), have received considerable attention from linguists and psycholinguists alike.

The prize that the ring that the jeweler that the man that she liked visited made won was given at the fair.

Such sentences are generally regarded as being extremely difficult to comprehend, even though grammatical, and much research has been devoted to demonstrating that they are more difficult to understand than other sentences of comparable semantic complexity but different structure, i.e., that there is a special difficulty involved in understanding self-embedded sentences.

Miller and Isard (1964), for example, studied the free recall of sentences of constant length but varying numbers of self-embedded relative clauses, finding that recall tended to become worse as the number of selfembeddings increased. Blumenthal (1966) found that subjects often paraphrased sentences with multiple self-embeddings incorrectly; and Stolz (1967) found that only after considerable practice could subjects interpret such sentences correctly. Foss and Lynch (1969) and Hakes and Foss (1970) showed that on-line comprehension difficulty, measured by the phoneme monitor task, was greater for doubly self-embedded sentences than for right-branching sentences. And more recently, Blaubergs and Braine (1974) have reported that in a short-term memory probe task sentences with three or more levels of self-embedding were retained more poorly than comparable right-branching sentences.

Given the evidence of these and numerous other

This research was supported by Grant OEG-0-72-3941 from the National Institute of Education. The authors wish to thank Donald J. Foss for his comments. Author Brannon is now at McNeese State University, Lake Charles, Louisiana 70601. studies, there seems little doubt that self-embedded sentences are difficult to understand and, in particular, more difficult than synonymous right-branching sentences. The question is how best to account for this difficulty.

The "standard" account is one proposed by Miller and his co-workers (see, e.g., Miller \& Chomsky, 1963; Miller \& Isard, 1964), based on the observation that self-embedded sentences like Sentence 1 are more difficult to understand than right-branching sentences like Sentence 2 that have the same number of relative clauses, and based also on formal considerations concerning how a sentence comprehension device might operate that made only a single left-to-right pass through a sentence.

She liked the man that visited the jeweler that made the ring that won the prize that was given at the fair.

Miller noted that each relative clause in a self-embedded sentence interrupts the clause in which it is embedded, something not true in a right-branching sentence. He proposed, essentially, that in comprehending a selfembedded sentence the processing of the main clause must be interrupted between the subject and predicate in order to process the first relative clause; processing of that clause must, in turn, be interrupted to process the second relative clause, and so on for as many clauses as are embedded. Assuming a limited capacity shortterm processing memory that holds partially analyzed parts of clauses until their completions are available, Miller argued that more than a few self-embeddings would overload the memory, resulting in comprehension difficulty and/or failure.

Since Miller proposed the "interruption" hypothesis, it has achieved nearly the status of a received truth, and its validity has rarely been challenged. Further, 
it seems to be equally widely accepted that the difficulty function for self-embedded sentences is discontinuous. One level of self-embedding is generally believed to cause little or no difficulty, while two or more levels cause considerable difficulty (see, e.g., Miller \& Chomsky, 1963).

Miller and Chomsky appear to have suggested such a discontinuity on the basis of informal intuitions; and it does seem intuitively clear that increasing the number of self-embedded clauses does increase difficulty and the likelihood of comprehension failure. But intuitions about difficulty seem to reflect most directly on the number of self-embeddings necessary to cause comprehension failure, and it seems clear that difficulty can vary considerably before reaching a level where it results in complete failure.

The results of Miller and Isard's study have often been taken as suggesting a difficulty discontinuity between one and two levels of self-embedding. These authors do note that across successive recall trials "the performance curves for zero and one degree of self-embedding are quite close, as are the curves for three and four embeddings, with performance on the doubly embedded sentence falling somewhere in between." But they present no analysis which would support the conclusion that the difficulty difference between one and two embeddings is significantly greater than that between zero and one. In any event, their data concern multiple-trial free recall; and there is no particular reason to believe that verbatim recall is related in any simple way to comprehensibility. In addition, as we will indicate below, all betweencondition comparisons in this experiment are confounded, with the result that their interpretation must be unclear.

We suggest, then, that there is little empirical reason to believe that comprehension difficulty increases discontinuously with the number of embeddings. Further, we note in passing that different estimates of where the discontinuity occurs do not agree. Conventional wisdom has it that the discontinuity occurs between one and two embeddings; Blaubergs and Braine, on the other hand, report a discontinuity in their probe recall data between two and three embeddings.

Our concem here is not to question the conventional wisdom that self-embedded sentences are difficult to understand. They are. Our concern is, rather, to question whether the interruption hypothesis accounts for that difficulty.

In comparing self-embedded sentences with rightbranching sentences, there is, as Miller noted, a difference in the location of the relative clauses. In the former, each relative clause modifies the subject of the clause that dominates it, separating the subject of that clause from the predicate. In the latter, each relative clause modifies the object of the dominating clause and occurs after that clause's predicate.

However, for the self-embedded and right-branching sentences that have been used in all the pertinent experiments there is an additional difference. These types of sentences have differed in the internal structure of the relative clauses used, a difference that has been completely confounded with the self-embedded vs. right-branching difference. The relatives used in the selfembedded sentences have been object relatives, ones in which the direct object of the clause has been fronted and relativized. Those used in the right-branching sentences, however, have been subject relatives, ones in which the subject of the relative clause has been relativized. Given this confounding of a difference in relative clause location with a difference in the internal structure of the relatives, it is entirely possible that the results of all the pertinent experiments could be attributed to the internal structure difference rather than to the difference between self-embedded and rightbranching locations.

Thus an unbiased evaluation of the interruption hypothesis requires breaking the confounding that has been present in previous studies of the difficulty of self-embedding. This could be accomplished in either of two ways: (1) holding the internal structure of the relative clauses constant while varying their location, or (2) holding their location constant while varying their internal structure. The first of these is the more direct test of the interruption hypothesis, and we have chosen it for that reason. Experiment I examines the selfembedded vs. right-branching difference using object relative clauses; Experiment II examines the same difference using subject relative clauses.

One problem associated with this approach is that the sentences used in the two conditions of each experiment cannot be synonymous. This, of course, is a common problem in research on sentence comprehension, for it is generally the case that differences in structure are associated with differences in meaning. In fact, some of the earlier, confounded studies of self-embedding and right-branching allowed meaning to vary between conditions (see, e.g., Foss \& Lynch, 1969). We have attempted to minimize the possible influence of such semantic differences in ways described below.

A second problem is more apparent than real. As we have noted earlier, it is generally believed that the difficulty of self-embedded sentences increases discontinuously with the number of such embeddings. Even though there is scant evidence for such a discontinuity, it is clear that difficulty does increase with the number of such embeddings. For this reason, the strongest comparison of self-embedded and right-branching sentences would be between sentences with several object relative clauses occurring in self-embedded 
position and sentences with the same number of object relatives in right-branching position (or, alternatively, between sentences with several self-embedded subject relatives and ones with an equal number of right. branching subject relatives).

Such a comparison is, however, impossible in principle. Sentence 3 contains a single right-branched object relative clause.

The girl hated the teacher that the boy liked.

Since that clause is verb final, there is no way in which that clause can be modified by another relative clause without its being interrupted, i.e., without the second clause being embedded within the first. A similar problem arises when using subject relative clauses, though here the difficulty occurs when such clauses are selfembedded rather than right-branched. Attempting to construct the relevant examples should quickly convince the reader.

The result of this is that the comparison must be between one level of self-embedding and zero, between two levels and one, etc. As we have indicated earlier, conventional wisdom to the contrary, there is no compelling reason to believe that the difficulty difference between two levels of self-embedding and one is any greater than the difference between one level and zero, so we have chosen the latter comparison to examine.

It does seem possible, however, that a difference in comprehension difficulty between self-embedded and right-branching sentences involving only single relative clauses might not become apparent unless the overall level of comprehension difficulty is relatively high. For this reason, another variable-relative clause reduction-was included in both experiments. Fodor and Garrett (1967) have shown that omitting the relative pronouns from doubly self-embedded sentences results in their being even more difficult to comprehend. Hakes and Cairns (1970) and Hakes and Foss (1970) have shown that this reduction also significantly affects performance on an on-line measure of comprehension difficulty, the phoneme monitor task. For this reason, each sentence in each experiment was presented with its relative clause either reduced or unreduced.

To assess comprehension difficulty, we chose two tasks: paraphrasing and phoneme monitoring. The first of these provides a postsentential measure of comprehension difficulty. The second requires that the subject, while trying to understand a sentence presented auditorily, listen in the sentence for a word beginning with a specified target phoneme and, if the target occurs, respond to it as quickly as possible. A number of experiments have shown reaction time in this task to be a sensitive measure of on-line comprehension difficulty attributable to sentence structure (e.g., Foss
\& Lynch, 1969; Hakes \& Cairns, 1970; Hakes \& Foss, 1970; Hakes, 1972), to ambiguity (e.g., Foss, 1970; Foss \& Jenkins, 1973; Swinney, 1974), and to a variety of other variables that have been hypothesized to affect comprehension difficulty.

Use of two comprehension difficulty measures allows us to test two aspects of the interruption hypothesis. First, that hypothesis predicts that sentences containing self-embedded relatives will be more difficult to comprehend than one containing right-branching relatives; such a difference should appear in a global measure of difficulty such as the paraphrasing task. Second, the hypothesis predicts that the difficulty difference between self-embedded and right-branching relatives should first manifest itself within the relative clauses themselves, for it is just the necessity of holding information about the interrupted embedding clause that is claimed to cause the greater difficulty of the former. Thus, measuring phoneme monitoring latency to targets within the relative clauses provides a test of whether a difficulty difference arises where a hypothesis predicts.

\section{EXPERIMENT I}

\section{Method}

Design and materials. Twenty sentences were constructed, each of which contained a single object relative clause which could modify either the subject (selfembedded) or object (right-branching) of the sentence's main clause, e.g., Sentence 4. The subject and object NPs of each sentence's main clause were interchangeable.

After the final curtain on opening night, the director (that) the repertory company had hired praised the star performer.

After the final curtain on opening night, the star performer praised the director (that) the repertory company had hired.

Thus, the content of the relative clause was the same, as was the NP it modified, regardless of whether it modified the subject or the object of the main clause.

Since each sentence could be presented with its relative clause either reduced or unreduced and with the relative clause modifying either the subject or object of the main clause, there were four versions of each sentence. The sentences were all complex in ways additional to the relative clauses, containing other main and subordinate clauses and/or complex modifiers.

Each sentence was constructed so that a word within the relative clause contained the target phoneme for monitoring. This was either the subject noun of the relative or a prenominal adjective modifying that noun. Thus, in Sentence 4 the target phoneme was the $/ \mathrm{r} /$ in repertory. The particular phoneme that served as the target varied across sentences, but in the sentence in which it was the target, it did not occur elsewhere in word initial position.

Twentyeight filler sentences were also constructed, each of a degree of complexity comparable to that of the test sentences, but without any particular structural constraints observed in their construction. Of these, 15 contained monitoring targets, located at widely varying points and occurring in diverse types 
Table 1

Phoneme Monitoring Speed and Paraphrasing Accuracy: Experiment I

\begin{tabular}{ccc} 
Condition & $\begin{array}{c}\text { Monitoring } \\
\text { Speed }\end{array}$ & $\begin{array}{c}\text { Paraphrasing } \\
\text { Accuracy }\end{array}$ \\
\hline Self-Embedded & & \\
Unreduced & 1.997 & .451 \\
$\quad$ Reduced & 1.811 & .514 \\
Right-Branching & & \\
$\quad$ Unreduced & 1.934 & .409 \\
Reduced & 1.720 & .460 \\
\hline
\end{tabular}

of words. The remaining 13 filler sentences contained no monitoring targets and were included to prevent the subjects' expecting such a target to occur in every sentence.

There was thus a total of 48 sentences, 20 of which contained object relative clauses. A single quasi-random order of the 48 sentences was constructed, and four tape recordings of the sentences in this order were made. The 20 test sentences were randomly divided into four sets of five sentences each. On any one tape, one set occurred in each of the four possible combinations of relative clause location (self-embedded vs. right-branching) and relative clause reduction (reduced vs. unreduced). The assignment of test sentence versions of tapes was by an ordered roll-over technique.

Tones of $1,000 \mathrm{~Hz}$ were recorded on a separate track of each tape, inaudible to the subjects and coincident with the onset of each target phoneme. These were used to start the timer used to measure monitoring latency. Each sentence was preceded on each tape by an instruction specifying the target phoneme for that sentence. For the filler sentences not containing monitoring targets, the instruction was to listen for a phoneme that did not occur in word-initial position anywhere in that sentence. Instructions for the phoneme monitor and paraphrase tasks and four practice sentences were also recorded and dubbed onto the beginning of each of the four tapes.

To summarize, the design included four groups, defined by the four tapes, i.e., by the assignment of test sentences to conditions. Within subjects, there were two values of each of two variables: relative clause location (self-mbedded vs. right-branching) and relative clause reduction (reduced vs. unreduced). The design was thus a 4 by 2 by 2 mixed factorial.

Procedure. Subjects were assigned to groups randomly; all were tested individually by the same experimenter. The instructions and sentences were presented binaurally through headphones at a comfortable listening level. The general instructions indicated that the subject was to try to comprehend each sentence and was to: (a) listen for a word in each sentence beginning with the sound specified by the instruction preceding that sentence; (b) respond by pressing a button as quickly as possible if such a word was heard; and (c) say in his own words, after the sentence was finished, what the sentence meant. The instructions explicitly discouraged verbatim repetition of the sentences, and, if necessary, subjects were further instructed on this point following the practice sentences. The four practice sentences were then presented, followed by the 48 test and filler sentences. The button used for the monitoring response was mounted on a panel angled toward the subject at $30 \mathrm{deg}$ above the horizontal plane. Subjects responded with whichever digit they preferred. Paraphrase responses were made orally and tape recorded for later analysis.

Subjects. Forty students from the freshman-level introductory psychology course at the University of Texas at Austin served as subjects. Participation in experiments was a course requirement, but the subjects were not required to participate in this particular experiment. All were native speakers of English, and none had previously served in any experiment on sentence comprehension. Approximately equal numbers of males and females served, though subjects were neither selected nor assigned to groups on this basis.

\section{Results and Discussion}

Phoneme monitoring. Individual trial monitoring latencies were converted to speed scores by reciprocal transformation. For each subject, four mean speed scores were computed, one for the sentences of each of the four conditions. The means of these mean speed scores, taken across groups, are presented in Table 1 and indicate that monitoring was faster when the relative clauses were self-cmbedded than when they werc rightbranching. Further, as anticipated, monitoring was faster when the relative clauses were unreduced than when they were reduced.

The mean speed scores were subjected to a Groups by Self-Embedded vs. Right-Branching by Reduced vs. Unreduced analysis of variance. The main effect for Self-Embedded vs. Right-Branching was significant, $\mathrm{F}(1,36)=5.499, \mathrm{p}<.05, \mathrm{MS}_{\mathrm{e}}=.437$, as was the main effect for Reduced vs. Unreduced, $F(1,36)=24.617$, $\mathrm{p}<.001, \mathrm{MS}_{\mathrm{e}}=.652$. The main effect for Groups was far from significant, $F<1$, and no interactions approached significance.

The effect attributable to relative clause reduction indicates that omitting the relative pronouns from object relative clauses substantially increased on-line processing difficulty even when the sentences upon which this variation was worked were less complex than the doubly selfembedded sentences used in earlier experiments.

The effect of main interest is, of course, that attributable to the self-embedded or right-branching location of the relative clauses, for the interruption hypothesis clearly predicts that monitoring should be slower for selfembedded clauses than for right-branching clauses. The results indicate, however, that monitoring was faster for the selfembedded clauses. The effect is small in magnitude, and, to determine its reliability, a second analysis was performed. Here the data were analyzed sentence by sentence, with the speed scores collapsed across groups. The effect for the Self-Embedded vs. Right-Branching comparison was marginally significant $(.05<\mathrm{p}<.10$, two-tailed Wilcoxon matched pairs signed-ranks test). (The effect for the Reduced vs. Unreduced comparison was highly significant, $\mathrm{p}<.01$.) Thus, whether analyzed by subject or by sentence, the results show much the same effect: Sentences with selfembedded object relative clauses yield marginally faster phoneme monitoring latencies than sentences with right-branching object relative clauses. However, whether this is a reliable effect or not is of only minor interest; for, even it if is not, the results are in the direction opposite to that predicted by the interruption hypothesis. Hence, there is no way these results can be interpreted as lending aid or comfort to that hypothesis. 
Paraphrasing. Each test sentence was divided into segments corresponding roughly to surface structure clauses, there being either three or four such segments per sentence. The paraphrase responses were scored on the basis of the number of such segments accurately represented in the response. To be scored correct, both the internal grammatical relations of a segment and that segment's relations to the sentence's other segments had to be represented accurately. Lexical substitution was liberally allowed, except for the substitution of an indefinite term for a definite one (e.g., someone for the director). Verbatim repetitions of a sentence were not credited.

Each paraphrase response was transcribed and was scored independently by two judges. Each judge scored all paraphrases of all versions of a given test sentence before proceeding to the next sentence. Differences in the scores assigned by the two judges were resolved by discussion (sometimes acrimonious) after all scoring was completed.

Since the number of segments scored varied across sentences, a subject's accuracy score was taken as the proportion of the sentence's segments paraphrased accurately. Four mean accuracy scores were then computed for each subject, taking the mean proportion correct across the sentences in each of the four conditions. The means of the mean accuracy scores, taken across groups, were presented in Table 1.

The mean accuracy scores were subjected to an analysis of variance. The main effect for Self-Embedded vs. Right-Branching was highly significant, $F(1,36)=7.653$, $\mathrm{p}<.01, \mathrm{MS}_{\mathrm{e}}=.120$. The effect here is thus consistent with that obtained for monitoring latency: Accuracy is greater for selfembedded object relatives than for rightbranching object relatives. Again, the effect is just the opposite of that predicted by the interruption hypothesis.

The main effect for Relative Clause Reduction was also highly significant, $\mathrm{F}(1,36)=10.067, \mathrm{p}<.01, \mathrm{MS}_{\mathrm{e}}=$ .128 . But, paradoxically, the effect was in the wrong direction: Paraphrasing was better when the relatives were reduced than when they were not. This result is consistent neither with the results obtained here for phoneme monitoring nor with previous paraphrasing results. The interaction of Relative Clause Reduction with Self-Embedded vs. Right-Branching location was not significant, $F<1$. The only other effect to reach significance was the Groups by Reduced vs. Unreduced interaction, $\mathrm{F}(3,36)=7.630, \mathrm{p}<.01, \mathrm{MS}_{\mathrm{e}}=.128$, indicating that, for one assignment of test sentences to conditions, paraphrasing was more accurate for the unreduced relatives while, for the other three assignments, paraphrasing was more accurate for the reduced relatives.

To investigate further the reliability of these effects, the paraphrasing accuracy data were also subjected to a sentence-by-sentence analysis. Here the effect for selfembedded vs. right-branching, although in the same direction as in the analysis by subjects, was not significant $(p>.05)$; the effect for reduced vs. unreduced was significant $(\mathrm{p}<.05)$, both by two-tailed Wilcoxon tests.

In summary, then, the results for both tasks are consistent in indicating that comprehension difficulty is no greater for sentences containing selfembedded object relatives than for sentences containing rightbranching object relatives. Whether difficulty is actually less for the selfembedded sentences is not entirely clear. But, since the interruption hypothesis clearly requires it to be greater, even a nonsignificant effect in the wrong direction is sufficient to disconfirm that hypothesis. The conventional wisdom that selfembedded sentences are more difficult to understand than almost anything else (including right-branching sentences) is clearly not supported.

\section{EXPERIMENT II}

Experiment I has demonstrated that, for object relatives, interruption of the dominating clause does not result in increased comprehension difficulty, thus disconfirming the interruption hypothesis. But that hypothesis does not apply just to object relatives. It claims, rather, that interruption of a dominating clause should result in increased comprehension difficulty regardless of the nature of the embedded clause. Thus the interruption hypothesis predicts the same outcome concerning selfembedded or right-branching clause locations for subject relatives as it did for object relatives: Self-embedded subject relatives should result in greater comprehension difficulty than the same relatives in right-branching location.

Experiment Il tested this prediction, comparing sentences containing subject relatives in either selfembedded or right-branching location.

\section{Method}

Design and materials. The materials here paralleled those of Experiment I as nearly as possible, the exception being the internal structure of the relative clauses used. Again, 20 test sentences were constructed, each of which contained a single subject relative that could modify either the subject or the direct object of the sentence's main clause, e.g., Sentence 5.

The children (that were) playing in the hayloft startled the farmer's wife when she went to gather the eggs.

The farmer's wife startled the children (that were) playing in the hayloft when she went to gather the eggs.

Whenever possible, the sentences of Experiment I were used, modified in the appropriate way.

The verb in the relative clause was always in the past progressive tense, allowing us to present the sentences with the relative clauses either reduced or unreduced. There is a general rule of English syntax which prohibits reducing a subject relative if its main verb is nonprogressive. One reason for this appears to be that, when such a relative clause is reduced, the fact of its 
Table 2

Phoneme Monitoring Speed and Paraphrasing Accuracy: Experiment II

\begin{tabular}{ccc} 
Condition & $\begin{array}{c}\text { Monitoring } \\
\text { Speed }\end{array}$ & $\begin{array}{c}\text { Paraphrasing } \\
\text { Accuracy }\end{array}$ \\
\hline Self-Embedded & & \\
Unreduced & 1.989 & .679 \\
Reduced & 2.021 & .646 \\
Right-Branching & & \\
Unreduced & 2.009 & .625 \\
Reduced & 1.984 & .628 \\
\hline
\end{tabular}

existence is obscured, making miscomprehension likely (see Bever \& Langendoen, 1971).

The filler sentences were identical to those of Experiment $I$, as were all details of tape construction and the placement of tones for phoneme monitoring. The monitoring target for each test sentence was the initial phoneme of the head noun of the relative clause's direct object. Here, as in Experiment I, there were four groups of subjects, defined by the assignment of test sentences to the self-embedded and right-branching conditions and the reduced and unreduced conditions. Within subjects, these four conditions were combined orthogonally.

Procedure. The procedure was identical in all respects to that of Experiment I.

Subjects. Forty subjects, having the same general characteristics as those used in Experiment I, participated. Ten were assigned to each group according to a quasi-random schedule.

\section{Results and Discussion}

Phoneme monitoring. Reduction of the monitoring data to mean speed scores was accomplished in the same manner as in Experiment $I$, with one exception. After data collection was completed, it was discovered that one test sentence had inadvertently been misrecorded on one tape. Data for this sentence were eliminated for all groups, with the result that the mean speed scores for the conditions into which that sentence entered were based on one fewer latency than the other conditions. The means of these mean speed scores, taken across groups, are presented in Table 2.

Ove iall, responding here was rather faster than in Experiment I. This suggests that the subject relatives caused less comprehension difficulty than the object relatives, though it might also reflect other differences between the sentences of the two experiments, e.g., the difference in monitor target location. The difference between the self-embedded and right-branching conditions is in the same direction as in Experiment $I$, monitoring being slightly faster when the relatives were self-embedded. The effect is, however, far smaller than that obtained with object relatives. Monitoring was also slightly faster when the relatives were reduced than when they were unreduced, but again the effect is miniscule.

Analysis of variance of the mean speed scores bears out the impressions gained by inspection. The main effects for Self-Embedded vs. Right-Branching and for Reduced vs. Unreduced were both far from significant, $F<1$ in both cases. The only effect to reach signifi- cance was that attributable to the Groups by SelfEmbedded vs. Right-Branching interaction, $F(3,36)=$ $3.880, \mathrm{p}<.02, \mathrm{MS}_{\mathrm{e}}=.067$. In the absence of a significant main effect for Self-Embedded vs. Right-Branching, this is of little consequence.

As in Experiment I, the monitoring data here were also analyzed sentence by sentence, with the speed scores collapsed across groups. The results were completely consistent with those obtained when the data were analyzed by subject: Neither the SelfEmbedded vs. Right-Branching comparison nor the Reduced vs. Unreduced comparison approached significance. It is perhaps worth noting, however, that for the former comparison the direction of the difference was the same as in the analysis by subjects: Monitoring tended to be faster when the relatives were selfembedded than when the same relatives were rightbranching in the same sentences.

It appears, then, that no substantial difference in on-line processing load resulted either from a difference in the selfembedded or right-branching location of the subject relatives or from whether they occurred in complete form or with the relative pronouns and auxiliary verbs deleted. The fact that the processing load was not greater when the relatives were self-embedded is, of course, inconsistent with the interruption hypothesis. Since no effects of interest were significant in the monitoring data for Experiment II, it might be argued that the design and/or materials used were not sufficiently sensitive to allow detection of a small difference of the sort predicted by the interruption hypothesis. The fact that significant differences were obtained using the same design in Experiment I (albeit not the one predicted by that hypothesis) argues against such a conclusion, as does the fact that, whether analyzed by sentence or by subject, the difference is in the wrong direction.

In addition, informal comparison of the present results with those of Experiment I suggests that subject relatives caused less on-line comprehension difficulty than object relatives, regardless of whether they were self-embedded or right-branching, a point to which we will return.

Paraphrasing. The paraphrasing data were treated in a manner identical to that described for Experiment I, with the same exception noted above for the phoneme monitoring data for Experiment II. Again, two judges independently scored each paraphrase, using the same procedures and criteria as in Experiment I.

The paraphrasing data are presented in Table 2 in terms of mean proportion accuracy scores, taken across groups, for the four conditions. The overall level of accuracy is considerably higher than that found in Experiment I for sentences containing object relatives, again suggesting that subject relatives cause less comprehension difficulty than object relatives. Accuracy appears to have been somewhat higher for subject rela- 
tives that were selfembedded than for the same relatives when they were right-branching, and also somewhat greater when the relatives were unreduced than when they were reduced.

Analysis of variance of the paraphrasing data revealed that the main effect for Self-Embedded vs. RightBranching was significant, $F(1,36)=4.503, p<.05$, $\mathrm{MS}_{\mathrm{e}}=.012$. The main effect for Reduction was not significant, $\mathrm{F}<1$. Somewhat complicating the interpretation of these results is the fact that the main effect for Groups was significant, $F(3,36)=3.133, p<.05$, $\mathrm{MS}_{\mathrm{e}}=.086$, as were the interactions of Groups by SelfEmbedded vs. Right-Branching, $F(3,36)=9.463$, $p<$ $.001, \mathrm{MS}_{\mathrm{e}}=.012$, and Groups by Self-Embedded vs. Right-Branching by Reduction, $F(3,36)=3.126, p<$ $.05, \mathrm{MS}_{\mathrm{e}} .014$. Inspection of the data suggests that these effects are primarily attributable to atypically low accuracy (.42) for one group of subjects for the sentences with reduced relatives in self-embedded location. Performance for that set of sentences under other conditions for other groups of subjects was not atypical, suggesting that the cause of the atypical performance in that one cell was not the sentences themselves. However, the subjects in the group contributing the atypical performance tended to be less accurate for all conditions than the subjects in the other groups, suggesting that it was the subjects that were atypical. Inspection of the phoneme monitoring data failed to yield any evidence of unusual performance for this group.

To check on the consistency of the effects obtained in this analysis, the paraphrase data were also analyzed by sentence in the manner described for Experiment I. Sentences with selfembedded subject relatives were paraphrased significantly more accurately than sentences with right-branching subject relatives $(\mathrm{p}<.05$, twotailed Wilcoxon test). The difference favoring greater paraphrasing accuracy for sentences with unreduced relatives was marginally significant $(.05<\mathrm{p}<.10$, two-tailed Wilcoxon test). Thus, the effect for relative clause location appears consistent across test sentences; and the reduction effect which was masked in the subject analysis by the presence of two significant interactions may be a real, though small, effect.

The paraphrasing data are, then, consistent with the monitoring data in showing no consistent effect for relative clause reduction. While there is some suggestion that such an effect exists and is being masked, it seems clear that it is not an effect of major importance. The paraphrasing data do, however, reveal a significant difference between self-embedded and rightbranching subject relatives that was not apparent in the monitoring data. Finally, the overall levels of paraphrasing accuracy for the two experiments suggest, as do the phoneme monitoring latencies for the two, that subject relatives cause less comprehension difficulty than do object relatives.
It is somewhat problematic that the paraphrasing and phoneme monitoring data for this experiment are not consistent: For the former, selfembedded relatives caused significantly less comprehension difficulty than right-branching, while, for the latter, the difference was not significant. It should, of course, be borne in mind that the discrepancy here concerns only whether selfembedded subject relatives are easier to process than right-branching ones or whether there is no difference; in neither case is there any suggestion that self. embedded clauses are harder to process.

We have no account of the difference in which we have any confidence. It does seem possible that for the sentences in question the monitoring targets were located at points at which the processing load differed only minimally and that locating them differently would have yielded a larger difference. Altematively, it is possible that subject relatives, regardless of where they are located, add little to the amount of on-line processing involved in initially comprehending a sentence. If so, the difference obtained with the paraphrase task might reflect some difference associated with the construction of paraphrases itself. The present data shed no light on which of these, or of the myriad other possibilities, might be correct.

\section{GENERAL DISCUSSION}

The relative clause reduction variable was included in the present experiments mainly to increase the overall comprehension difficulty of the sentences. We had anticipated that difficulty differences between selfembedded and right-branching sentences might appear only if overall difficulty was high. Clearly, this anticipation was disconfirmed, for in neither experiment was there an interaction between reduction and the selfembedded vs. right-branching difference. Whatever differences in difficulty resulted from these two manipulations were quite independent.

The results of main interest are, of course, those concerning the difference between selfembedded and right-branching relative clauses. The results seem clear and unambiguous: When the internal grammatical structure of the relatives is controlled, there is no evidence whatsoever that sentences containing selfembedded relatives are more difficult to comprehend than ones containing right-branching relatives. There is, in fact, some suggestion that the latter are the more difficult. Given the present evidence, the interruption hypothesis cannot be correct.

However, a question remains. If sentences containing self-embedded relatives are not more difficult to understand than ones containing right-branching relatives, then how are we to account for the results of all those experiments that have concluded the contrary? Recall that in all of those experiments the comparison was between selfembedded object relatives and right. 
branching subject relatives. If the greater difficulty of the former cannot be attributed to the fact of selfembedding, then it seems to follow logically that the difficulty is attributable to the difference between subject and object relatives.

This conclusion would be more palatable if, in addition to a logical argument, we could support it with a direct empirical argument. Certainly, a comparison of the results of Experiments I and II is suggestive, for it does appear that for both tasks object relatives are more disruptive of comprehension processing than are subject relatives, regardless of whether those clauses are selfembedded or right-branching. We are, however, reluctant to place great confidence in such a betweenexperiments comparison. Neither the sentences nor the monitoring targets are directly comparable for the sentences of the two experiments. In addition, the data for the two were collected from different subjects at different times by different experimenters. We have, for these reasons, refrained from performing any statistical analysis of the betweenexperiments comparison.

We should note in closing, however, that Fodor, Bever, and Garrett (1974, see especially pp. 344-346) present an argument, together with some supporting data, rationalizing the conclusion to which our experiments seem to point: Object relative clauses are more difficult to understand than subject relative clauses; all other things equal, self-embedded relatives are not more difficult than right-branching relatives.

\section{REFERENCES}

Bever, T. G.. \& Langendoen, D. T. A dynamic model of the evolution of language, Linguistic Inquiry, 1971, 2. 433-464.

Blaubergs, M. S.. \& Braine, M. D. S. Short-term memoty limitations on decoding self-embedded sentences. Journal of Experimental Psychology, 1974, 102, 745-748.

Blumenthal, A. L. Observations with self-embedded sentences. Psychonomic Science, 1966, 6, 453-454.

Fodor, J. A.. Bever, T. G.. \& Garrett, M. F. The psychology of language. New York: McGraw-Hill, 1974.

Fodor, J. A.\& GARRETr, M. Some syntactic determinants of sentential complexity. Perception \& Psychophysics, 1967. 2. $289-296$.

Foss, D. J. Some effects of ambiguity upon sentence comprehension. Joumal of Verbal Learning and Verbal Behavior. 1970, 9, 699.706.

Foss, D. J.. \& Jenkins, C. M. Some effects of context on the comprehension of ambiguous sentences. Journal of Verbal Learming and Verbal Behavior, 1973. 12. 577.589.

Foss, D. J.. \& LYNCH, R. H., JR. Decision processes during sentence comprehension: Effects of surface structure on decision times. Perception \& Psychophysics, 1969, 5, 145-158.

HAKES, D. T. Effects of reducing complement constructions on sentence comprehension. Journal of Verbal Learning and Verbal Behavior, 1972, 11, 278-286.

Hakes. D. T., \& Cairns. H. S. Sentence comprehension and relative pronouns. Perception \& Psychophysics. 1970, 8, 5-8.

HakEs, D. T., \& Foss, D. J. Decision processes during sentence comprehension: Effects of surface structure reconsidered. Perception \& Psychophysics, 1970. 8, 413-416.

Miller, G. A.. \& Chomsky, N. Finitary models of language users. In R. D. Luce, R. R. Bush, \& E. Galanter (Eds.), Handbook of mathematical psychologv. Vol. II. New York: Wiley, 1963. Pp. 419-492.

Miller, G. A., \& ISARD, S. Free recall of selt-embedded English sentences. Information and Control, 1964. 7, 292-303.

Srolz, W. S. A study of the ability to decode grammatically novel sentences. Journal of Verbal Learning and Verbal Behavior, 1967, 6. 867-873.

Swinney, D. A. Effects of context upon the processing of lexical ambiguities during sentence comprehension. Unpublished PhD dissertation. University of Texas at Austin. 1974.

(Received for publication June 5, 1975; revision received October 13,1975 ; accepted October 20. 1975.) 